\title{
37. COMMISSION DES AMAS STELLAIRES
}

\section{Président: M. H. Shapley, Director of the Harvard College Observatory, Cambridge 38 ,}

Mass., U.S.A.

Membres: MM. Bok, Chandrasekhar, Cuffey, Hertzsprung, Mme Sawyer Hogg, MM. N. U. Mayall, Mineur, Nassau, Trumpler, Vanderlinden, Wallenquist.

Unlike the investigations in many fields of astronomy, the work on star clusters offers few problems for practical international or inter-observatory co-operation. But there are two useful projects that the International Commission can undertake: the reporting of the researches in progress at the various observatories, and the co-operative listing of useful categories of research on star clusters. The list may serve as a guide for new workers in the field. Also there is, of course, much value in the mutual discussion of researches. Such interchange of ideas may be the most useful outcome of the Commission's conferences at the Zürich meeting of the International Astronomical Union.

Our preliminary and final report can be brief because surveys and bibliographies are being currently printed elsewhere. Mrs Helen Sawyer Hogg has completed for publication, from the David Dunlap Observatory, A Bibliography of Individual Globular Clusters, which will be of the utmost importance to all workers in the field henceforth. She expects to publish in a year or two a second edition of her catalogue of variable stars in globular clusters (originally published in 1939). The President of the Commission is preparing for publication in Popular Astronomy a generalized survey of work on globular star clusters during the past half century. Perhaps someone should undertake a similar bibliography and general survey for galactic clusters.

The members of the Commission have agreed that the following are the major categories of research on star clusters:

A. Open Clusters of the Milky Way System. (Here called galactic clusters)

I. Photometry in two or more colours and spectrum classification of individual stars.

2. Proper motions and radial velocities of galactic clusters and of the stars within galactic clusters, followed by studies of rotation and shear. Professor Hertzsprung emphasizes the importance of preparing, with great care, the first positions for stars in many clusters for studies such as have now been made for the Hyades, Pleiades, Praesepe, and Ursa Major; plate deterioration and magnitude equations must be guarded against.

3. Space structure and dynamics of the clusters (density distribution, stability, evidences of equipartition, etc.), and relation of galactic clusters to the Galaxy and its spiral arms.

4. Colour excesses, combined with photometry and spectrum classification, for estimates of distances and dimensions, and derivation of luminosity frequencies.

\section{B. Globular Star Clusters}

I. Photometry in the globular clusters of the galactic system, with special reference to colour-magnitude arrays and general luminosity curves. "For one-third of accepted globular clusters, no magnitudes are known.' (Mrs Sawyer Hogg.)

2. Continued studies of variable stars in and near globular clusters, including further investigation of period changes.

3. Space structure and dynamics (density distribution, flattening, etc.), both observational and theoretical.

4. Radial velocities (and eventually proper motions) of globular clusters, and of the brighter stars in globular clusters.

5. Colour excesses, and revision of distances for globular clusters in low latitudes.

6. Spectra (and colours) of giant stars in nearest globular clusters.

7. Intensive search for additional globular clusters. 


\section{Integrated Apparent Magnitudes of All Clusters (Globular and Galactic) in Various Colours. (Wallenquist.) \\ D. General Investigations of the Galactic and Globular Clusters of the Magellanic Clouds, the Andromeda Nebula, and Other Nearby External Galaxies.}

Dr Nassau suggests that the establishment of magnitude sequences for the work on clusters might be undertaken co-operatively.

The word photometry in the foregoing list is meant to include photographic, photoelectric, and radiometric methods.

It is proposed that brief statements of star-cluster work in progress at the various observatories will be presented at the Zürich meeting, and, together with summaries of work in two or three restricted fields, will be offered as an appendix to the present report.

HARLOW SHaPLeY President of the Commission

\section{Variable Stars in Globular Clusters}

\section{By Helen Sawyer Hogg}

Considerable information has been added to our knowledge of variable stars in globular clusters since I938. The publication in I939 of $A$ Catalogue of III6 Variable Stars in Globular Star Chusters (Sawyer, David Dunlap Publ. no. 4) served to put the subject in a systematic form. It is hoped to issue a second edition of this work about r950.

At present, 62 globular clusters have been systematically searched for variables, and the number known now in these objects is 1307 . The majority of these stars, well over a thousand, are RR Lyrae type variables; periods have been determined for about 800 of them. About 80 stars have been shown to have periods greater than one day, though a number of these are not actual cluster members.

Clusters in which new variables have been found since 1938 include the following: NGC 5634 (Baade), 6093 (Sawyer), 6r7x (Oosterhoff), 6229 (Baade), 6273 (Sawyer), 6284 (Sawyer), 6287 (Sawyer), 6293 (Sawyer), 6366 (Sawyer), 6656 (Sawyer), 6779 (Sawyer, Rosino).

Clusters in which new periods have been published since r938 are: NGC 288 (Oosterhoff), 3201 (Wright), 4590 (Popper), 4833 (Wright), 5053 (Sawyer), 5 I39 (Martin), 5466 (Sawyer), 6I2I (A. de Sitter, Oosterhoff), 6205 (Sawyer, Kollnig-Schattschneider), 6218 (Sawyer), 6254 (Sawyer), 634I (Nassau, Hachenberg), 6656 (Sawyer), 67I2 (Oosterhoff), 6779 (Sawyer, Rosino). Clusters in which more or less extensive period revision and correction have been accomplished are: 5024, 5272, 5904 and 7078 .

Clusters which have been under investigation for a long while and for which more data ought to be published soon are: 6402,6934 and 7006 .

From the mass of individual detail, star to star and cluster to cluster, certain significant facts have arisen from the work of the past decade. These may be briefly summarized as follows:

(I) RV Tauri variables are now shown to be present in relatively large numbers as compared with the known galactic variables of this class. Special attention should be called to the importance of Joy's spectrographic programme for these stars, and others with periods greater than one day.

(2) Most of the typical Cepheids found are of the W Virginis type, with periods just over two weeks.

(3) There is a continuing absence of eclipsing variables and novae, and great rarity of long-period variables physically associated with the cluster.

(4) The distribution of types of variable stars found in globular clusters indicates that the population of these clusters is Type II as defined by Baade. 
(5) The distribution of frequency of short periods is curious, with single or double maximum of the frequency curve, as discussed by Oosterhoff and Sawyer.

(6) There may be more very short period RR Lyrae stars than previously recognized in clusters (6205, Kollnig-Schattschneider, 6333, Sawyer, unpubl.).

(7) Extensive investigation of period changes shows no uniform pattern; periods are found to be increasing, decreasing, or remaining relatively constant.

Some of the problems to be stressed for future investigations are:

A. To establish good magnitude sequences over large intervals, sufficient to measure the very bright variables and the faint RR Lyrae type with equal accuracy.

B. The determination of the light curves of the RV Tauri and other variables with periods of some weeks, where dozens of nights, as nearly consecutive as possible, are necessary. The scale of the instrument must be sufficiently large to disentangle these stars from the background, but not so large that bright comparison stars are not nearby on the plates.

C. Research for variables in the highly obscured clusters. For some of the badly obscured clusters like NGC 640I, where it is suggested that even with the largest telescopes photographs must be taken in the red to reach the cluster stars, the problem of detecting variables and then determining their light changes seems to be a very serious one.

D. It is hoped that results on new or old variables will be published in a standard form; that authors will consult already existing lists and avoid renumbering known variables (as is done too frequently), thus causing an unnecessary amount of confusion in the literature. It is suggested too, that to conform to the homogeneity of the literature on the subject, authors publishing period determination work, publish an epoch of maximum (or minimum), and the actual value of the period.

\section{Spectral Classes and Colour Indices in Galactic Star Clusters}

\section{By Robert J. Trumpler}

The observational material on spectral classes and colour indices in galactic star clusters published during the last ro years is not very extensive.

It seems, however, appropriate at the present moment to review and discuss the most promising points of attack in this field and the kind of data most needed for further progress. The principal lines of investigation for which spectral classes and colour indices in galactic star clusters are useful are:

(I) The determination of distances from magnitudes and spectral classes.

(2) The study of colour excess and its relation to interstellar absorption.

(3) The luminosity-spectral class distribution, or the population characteristics of galactic clusters.

(4) The study of spectral peculiarities.

From magnitudes and spectral classes of its stars the distance modul $y=m-M$ of a galactic cluster can be found with a fair degree of accuracy. To derive the distance from the distance modul it is necessary to allow for the effect of interstellar absorption. In view of the irregular distribution of interstellar matter the assumption of uniform absorption leads to considerable errors in the distances of individual clusters, particularly the more remote ones. A more reliable estimate of the absorption effect could probably be obtained by assuming it proportional to the colour excess. More extensive and more accurate data on colour excess in galactic star clusters are urgently needed. They serve not only for improved distance determinations, but are also of interest for studying the distribution of absorbing matter. Moreover, galactic clusters offer an opportunity for finding the average ratio of colour absorption to general photographic (or visual) absorption.

For work on colour excess both spectral classes and colour indices must be determined for the same stars; the list of stars observed in a cluster, however, does not need to be 
complete nor very large. It is more important to observe a few stars (ro-20) in each cluster, and to investigate a whole series of clusters (including near and distant objects) with uniform equipment and procedure. The principal difficulty at present lies in the lack of homogeneity of colour index data due to systematic differences depending on the magnitude and to differences of colour equation.

A recent study by C. Baum (unpublished thesis at the University of California, Berkeley) compares many of the magnitude and colour index measures in galactic clusters with each other. It finds the colour index series determined by Zug at the Lick Observatory by the half-filter method to be affected by appreciable systematic errors. The half-filter method, like the method of effective wave-lengths, requires laborious calibration and careful study of the peculiarity of the instrumental equipment used. When the derivation of colour excess is the main purpose, it seems therefore that comparisons with the North Polar Sequence (or other well-established photometric standard areas) are the most desirable procedure. They generally permit also a determination of colour equation and reduction to the international colour system. In such comparisons the precautions and reduction methods proposed by Blaauw and Weaver (AP. J. ro6, 366, I947) should be used in order to avoid systematic errors in the magnitude scales.

Baade's work on the two principal population types in stellar systems has brought renewed interest to the differences in luminosity-spectral class distributions of galactic star clusters which may be considered as variations of Baade's type I. Progress in this field has been made mainly through more detailed investigations of the nearer representatives of various cluster types. For such detailed studies the elimination of background stars is essential. Differential proper motion measures (van Maanen, Ebbighausen) on photographs taken with long-focus telescopes have now reached an accuracy which in many objects of moderate distance permits selection of cluster members with fair reliability. Radial velocity measures also have proved useful for this purpose at least for the brighter stars in a cluster. Some of the features brought out, such as:

(i) the absence of ordinary giants of classes $\mathrm{F}-\mathrm{M}$ in clusters containing early B-type stars,

(ii) the occasional presence of super giant stars in such clusters but in no others,

(iii) differences among clusters in the central line or trend of the Main Sequence, seem highly important and should be more completely verified.

Spectroscopic observations of stars in galactic star clusters made with slit spectrographs in connection with radial velocity measures have shown that certain spectral peculiarities (Hydrogen emission, narrow or weak Hydrogen lines in class B stars; abnormal intensity of Ca III lines in classes A-F, etc.) often predominate or occur in considerable number among the members of the same group. Such clusters furnish a rare opportunity for studying the relation of peculiar spectra to normal spectra of the same class as well as connections between spectral peculiarities and population types. This appears to be a promising field for exploration which so far has been barely touched.

\section{The Dynamics of Globular Clusters}

\section{By Ivan KInG}

Progress since 1938 has been greater in some phases of globular-cluster dynamics than in others. The most rapid strides forward have been towards an understanding of the evolution of the clusters. Of primary importance was the recognition of the fact that as a result of exchanges of energy between individual stars of a cluster some members of the cluster will acquire sufficient energy to escape from its gravitational attraction. Discussions by Ambazrumian, Spitzer and Chandrasekhar have shown that a globular cluster may be expected to eject half its stars in a time of the order of $10^{11}$ years. Of great significance for future work is the setting up by Chandrasekhar of the mathematical mechanism, based on a generalization of the Liouville equation to include the effect of stellar encounters, necessary for a more exact and detailed treatment of cluster evolution. 
Closely linked with the problem of evolution is the observational determination of density distributions in clusters. Here the difficulties inherent in the photometry of crowded images are no less forbidding than in the past. Until a way is found to foil crowding and Eberhard effect-perhaps by direct photoelectric measurements-we can hope for little information about the highly interesting central regions of the clusters. Star counts in the outer parts, however, have been made in the past decade for Messier 92 by Hachenberg and Messier 4 by Greenstein. There is still need of more measurements of the relative density distributions at different magnitudes, with particular attention to the extended envelopes of faint stars. And star counts could well be supplemented by photoelectric tracings of surface brightness in the outer parts of clusters.

Studies of cluster dynamics will eventually have to consider the ellipticity of a cluster and its relation to the rotational motion (or perhaps its relation to encounters with other stellar groups). Although the exact problem is a forbidding one, it may be hoped that it will be possible within a few years to describe approximately the change of some suitably defined ellipticity parameter during the evolution of the cluster. New observations or new discussions of old observations of ellipticity have been few; worthy of mention is an attempt by Freundlich to correlate the ellipticity with the relative size of the envelope of faint stars about a cluster.

The application of the theory of cluster dynamics requires a knowledge of the masses of the clusters. Now that we have at our disposal accurate measurements by Christie of the total luminosities of the northern clusters, it is discouraging to realize that we cannot state with any degree of certainty the mass of a single globular cluster. Popper's recent measurements of radial velocities in Messier $\mathrm{I}_{3}$ indicate a mass for that cluster of not more than IO $^{5}$ solar masses; these measurements, however, were made on plates not well suited for the job, since the radial-velocity measurements were only a by-product of another observing project. Those astronomers having access to spectroscopic equipment on a large reflector should give the confirmation and extension of these measurements the highest priority, not only for the sake of cluster dynamics but also because of their importance to the general problem of stellar populations. Such a programme could well include an attempt to determine the order of magnitude of the rotational velocity of the cluster as well.

Another stumbling-block to practical applications of dynamical theory is our ignorance of the number of stars of small mass in a globular cluster. Since these stars are too faint to be photographed individually, the only way to estimate their number seems to be to subtract the total light of all stars photographed from the integrated light of the cluster. The accuracy of photographic photometry has proved inadequate for these delicate measurements, but the improved accuracy of the coming photoelectric age promises to bring them within the range of feasibility.

\section{The Progress of Galactic (Open) Cinster Dynamics Since ig38}

\section{By Uco vaN Wijk}

Substantial progress in the field of cluster dynamics was made during the period covered by this report. Our knowledge of the dynamics of galactic clusters under the influence of galactic shearing forces has been greatly extended by the work of Mineur, who found a relation between the shape and the density of a galactic cluster in statistical equilibrium, leading to a value of 0.6 solar masses per cubic parsec as the minimum density required for stability. In the same paper Mineur also made a thorough study of the distribution of the internal motions in galactic clusters. His work has been extended by Mayot who, in his doctoral thesis, considered the case of clusters which deviate slightly from a circular orbit around the centre of the Galaxy. A significant step forward in the correlation between theoretical studies and the observed shapes of clusters is the proof by Mayot that strongly elongated clusters are unstable. 
The minimum density required for the stability of galactic clusters as found by Mineur has led to a reconsideration of the existence of the very loose clusters of A stars. Gliese, Vyssotsky and Mrs Gossner have all analysed the available data for the extended Ursa Major cluster and the extended Taurus cluster. It was pointed out that the space velocities of these two groups lie close to the vertices of Kapteyn, blending gradually into the distribution of the space velocities of all A stars as a whole. The existence of these extended clusters as isolated dynamical units may therefore be considered doubtful. The loose B-star clusters in Scorpio-Centaurus and in Perseus were the subject for a similar investigation by Smart, who cast considerable doubt on their existence as clusters. On the other hand, the existence of the Scorpio-Centaurus cluster has been ably defended by Blaauw, but some difficulties are still encountered in the past evolution of this cluster.

The most important point in connection with the evolution of clusters is the rate of escape of stars from the clusters, which has been studied by Spitzer and by Chandrasekhar. Although their work was primarily intended for globular clusters, their results are also applicable to the denser galactic clusters. In an unpublished paper, Van Wijk has obtained a relation which allows a direct comparison between the influence upon cluster evolution of the loss of mass due to escape of stars and of the gain in total energy of the cluster due to encounters with field stars; he also generalized Mineur's relation between density and shape of galactic clusters.

The identification of faint members by their proper motion is of course an essential step in the advancement of our knowledge of the structure of galactic clusters. This type of work has been carried out by Van Maanen who identified a considerable number of stars as members of several well-known clusters. Of great value are measurements of the dispersion in the proper motions of the stars in a cluster, such as were obtained by Hertzsprung and by Titus for the Pleiades and by Schilt and Titus for Praesepe. Researches of this nature should be extended to other clusters when better proper motion data will become available.

Valuable data regarding the structure of the more remote galactic clusters can be obtained from star counts along the lines of the work of Zug and Vogt, and it would be desirable to continue these researches using the high image quality of modern instruments now available.

A survey of the field of cluster dynamics up until r942 is to be found in Principles of Stellar Dynamics by Chandrasekhar.

Report of meeting. Friday, August I3. I0.30-II.30 a.m.

President: Dr H. Shapley.

SeCretary: Dr R. J. TRUMPler.

Members of Commission PResent: Shapley (Chairman), Hertzsprung, Mineur, Nassau, Trumpler, Vanderlinden, Wallenquist.

The Chairman stated that in addition to the report published in the Draft Reports he had at his solicitation received several reports on special problems or on work in progress. He suggested that these be included in the Commission report and that they be briefly discussed at the meeting.

A summary of the Report by Mrs Helen Sawyer Hogg on Variable Stars in Globular Clusters was presented by the chairman, who also drew the attention of the Commission to the complete bibliography on globular clusters prepared by Mrs Sawyer Hogg. $\mathrm{He}$ suggested that a similar bibliography for galactic clusters would be very useful.

Trumpler outlined the principal points of his report on Spectral Classes and ColourIndices in Galactic Star Clusters.

Wallenquist elaborated on his suggestion for the determination of integrated apparent magnitudes in various colours; he advocated the use of photoelectric measures for this purpose. 
Nassau explained that his suggestion for the establishment of magnitude sequences for work on clusters has mainly the purpose of obtaining greater uniformity in magnitudes. He would like to have the observers publish the sequences used in each case.

Shapley drew attention to the value of Küstner's early position measures in several star clusters for the determination of proper motions, and recommended to Dr F. Becker, Director of the Bonn Observatory, the repetition of Küstner's early plates.

In connection with the report by Ivan King on the Dynamics of Globular Clusters, Shapley drew attention to the importance of gaining more information on the luminosity distribution in globular clusters. A comparison of integrated light with the total light of observed individual stars does not seem to leave room for large numbers of dwarf stars. The cluster $\omega$ Centauri seems to be a favourable object for studies of the luminosity distribution, and is now being investigated at the Harvard Observatory.

A report prepared by Uco van Wijk is concerned with recent progress in the dynamics of galactic clusters.

The programme of radial velocity measures in galactic star clusters undertaken by Trumpler is now nearing completion, and a report on this work is published by Commission 30 .

No resolutions were proposed to the General Assembly. 